PERCEPCIÓN DE ALIMENTOS EN EL PRIMER LUSTRO DE VIDA: ASPECTOS INNATOS, CAUSALIDAD Y MODIFICACIONES DERIVADAS DE LA EXPERIENCIA ALIMENTARIA FOOD PERCEPTION IN THE FIRST LUSTRUM OF LIFE: INNATE ASPECTS, CAUSALITY AND MODIFICATIONS DERIVED FROM REPEATED EXPERIENCIES WITH FOOD

Volumen 11, Número 3

Setiembre-Diciembre pp. 1-35

Este número se publicó el 15 de diciembre de 2011

Alejandro Chacón Villalobos

La revista está indexada en los directorios:

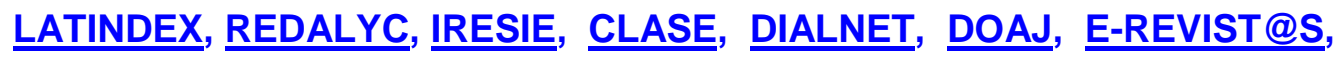

La revista está incluida en los sitios:

REDIE, RINACE, OEI, MAESTROTECA, PREAL, HUASCARAN, CLASCO 


\title{
PERCEPCIÓN DE ALIMENTOS EN EL PRIMER LUSTRO DE VIDA: ASPECTOS INNATOS, CAUSALIDAD Y MODIFICACIONES DERIVADAS DE LA EXPERIENCIA ALIMENTARIA
}

FOOD PERCEPTION IN THE FIRST LUSTRUM OF LIFE: INNATE ASPECTS, CAUSALITY AND MODIFICATIONS DERIVED FROM REPEATED EXPERIENCIES WITH FOOD

\begin{abstract}
Alejandro Chacón Villalobos ${ }^{1}$
Resumen: Este trabajo de revisión bibliográfica describe el concepto de la percepción sensorial innata de los niños y niñas hacia los alimentos, así como los procesos por medio de los cuales esta se modifica y enriquece a través de la interacción y las experiencias alimentarias durante el primer lustro de vida. Además, la importancia del marco afectivo y social en el que este proceso formativo se da. Tanto los espacios y contextos donde se consumen y se promocionan los alimentos durante la edad preescolar, el grado de disciplina impuesta, y el nivel de autodeterminación permitido a los menores tienen, en conjunto, un impacto que perdura a lo largo de toda la vida del ser humano. Por ello, la adecuada gestión de las experiencias sensoriales orientadas a generar improntas positivas en el hogar y en el aula, es de gran importancia para educadores y padres de familia por igual. La neofobia y la aversión a los alimentos, así como el rol de los medios de comunicación, son también abordadas complementariamente.
\end{abstract}

Palabras clave: ACEPTACIÓN DE ALIMENTOS, PERCEPCIÓN, NEOFOBIA, INSTRUCCIÓN, APRENDIZAJE, PREESCOLAR

\begin{abstract}
This paper review describes the innate sensorial perception of the children towards foods, as well as the processes by means of which this perception modifies and enriches through the interaction and the exposure to food during the first five years of life. It evaluates, in addition, the importance of the affective and social frame in which this formative process occurs. The spaces and contexts in which the foods are both consumed and promoted, the degree of imposed discipline, and the level of self-determination allowed to the minors, have altogether an impact that lasts throughout the entire lifespan of the human being. For this reason, a suitable management of the sensorial experiences oriented to generate positive impressions at the home and at the classroom, is of great importance for both educators and parents. Food neophobia and aversion, as well as the roll of mass media are also explained complementarily.
\end{abstract}

Keywords: FOOD ACCEPTANCE, PERCEPTION, NEOPHOBIA, INSTRUCTION, LEARNING, PREESCHOOLER

\footnotetext{
1 Magister en Ciencias de Alimentos. Licenciado en Tecnología de Alimentos. Ambos títulos de la Universidad de Costa Rica. Investigador de la Estación Experimental Alfredo Volio Mata. Profesor de la Escuela de Zootecnia y de la Escuela de Tecnología de Alimentos. Todas las unidades anteriores pertenecientes a la Facultad de Ciencias Agroalimentarias de la Universidad de Costa Rica.
}

Dirección electrónica: alejandro.chacon@ucr.ac.cr

Artículo recibido: 15 de julio, 2011

Aprobado: 10 de noviembre, 2011 


\section{Introducción}

Actualmente, es reconocido que los niños y los adultos difieren grandemente en la forma en que perciben y aceptan los alimentos. Este contraste es causado no sólo por la menor experiencia con los alimentos por parte de los menores, sino también por una acentuada diferencia en la forma en que operan en ellos los sentidos, particularmente, el gusto y el olfato (Grenville et al., 2004; Menella et al., 2005). Esto explica el hecho de que muchos niños rechacen alimentos por razones que en muchas ocasiones son incomprensibles para los adultos, mientras que en otros momentos son propensos a ensayar combinaciones que no serían atractivas para sus padres o encargados, como por ejemplo, salchichas con chocolate o de guisantes (Pisum sativum) con jarabe de maple mencionados por Kuntz (1996).

Desde el nacimiento y durante los primeros años, cuando los sentidos son inmaduros, aspectos como la forma, la textura y hasta el color pueden jugar un rol más importante como componentes de la percepción sensorial de lo que representan en el caso de los adultos (Rodríguez, 2001). En los niños y las niñas, las sensaciones que emergen de la integración de la visión, el gusto y el olfato con la experiencia mecánico-táctil son de suma importancia en el establecimiento del agrado por los alimentos (Álvarez et al., 2003).

La formación de las percepciones y consecuentes preferencias sensoriales en el primer lustro de vida es un proceso multivarial, complejo y no del todo comprendido (Saint John Alderson y Ogden, 1999). Más allá de los sentidos, la percepción es influenciada por aspectos intrínsecos y extrínsecos, tales como el estado emocional de los infantes, el entorno sociocultural en que se desenvuelven (Popper y Kroll, 2003), el desarrollo social y cognitivo, la capacidad de aprendizaje, la coordinación motora, el estilo de crianza en el hogar, y las interacciones en el salón de clases con maestros y compañeros (Birch, 1999; Álvarez et al., 2003). Aspectos como los valores, los estereotipos, los prejuicios, las experiencias y las ideas hacen de la percepción un proceso muy individual y social a la vez (Bravo, 2004; Cartín, 2005).

Los espacios y contextos en que se consumen y promocionan los alimentos durante la edad preescolar, el grado de disciplina impuesta, y el nivel de autodeterminación permitido a los menores tienen en conjunto, con las determinantes sensoriales, un impacto en la aceptación y consumo de los alimentos que perdura a lo largo de toda la vida. Por ello, la 
adecuada gestión de las experiencias orientadas a generar improntas positivas en el hogar y en el aula es de gran importancia para educadores y padres de familia por igual.

\section{Desarrollo de la precepción sensorial hacia los alimentos}

\subsection{Concepción y vida intrauterina}

En el momento de la concepción existe poca evidencia de que las preferencias sensoriales de un ser humano estén genéticamente predeterminadas y que, en consecuencia, el genotipo sea más importante que el historial de vida y las experiencias (Birch, 1998; Birch, 1999).

Las diferencias genéticas entre los diferentes grupos raciales son, si acaso, responsables de muy poco de la variabilidad total en las preferencias alimenticias de un menor, predominando los factores asociados al ambiente sociocultural (Rozin y Fallon, 1986; Birch, 1999; Cullen et al., 2000). Esto justifica que no tenga ninguna razón de ser el evaluar los efectos genotípicos en la formación de las preferencias de los niños (Menella et al., 2005). El género, asimismo, no es una fuente de variación importante en la aceptación y en la preferencia de los alimentos en edades anteriores a la adolescencia (Temple et al., 2002; Wesslen et al., 2002; Grenville et al., 2004).

En todos los seres humanos parecen existir una innata preferencia por el sabor dulce, un rechazo por los sabores amargos y por los agrios, así como una indiferencia inicial al sabor salado (Álvarez et al., 2003; Cooke, 2004; Liem y De Graff, 2004); aspectos que están genéticamente predeterminados y que no son en definitiva aprendidos (Birch, 1999; Bartoshuk, 2000; Benton 2003). La predisposición por el sabor dulce, que suele empezar a disminuir en la adolescencia (Popper y Kroll, 2003), puede ser instintiva y estar orientada a aumentar la palatabilidad de la leche materna que es naturalmente dulce debido a la lactosa y en menor grado a las caseínas (Álvarez et al., 2003). Además, el sabor dulce suele asociarse en edades muy tempranas con fuentes de calorías muy disponibles mientras el amargo se considera como un indicador de toxicidad (Westenhoefer, 2002). Las disoluciones dulces también han demostrado reducir el dolor en infantes funcionando como calmantes (Pepino y Mennella, 2005).

Otro aspecto que parece estar determinado innatamente es la preferencia por alimentos de alta densidad energética (Escobar, 1999), presentándose incluso un desarrollo más lento de las preferencias por alimentos poco energéticos (Jansen y Tenney, 2000; 
Álvarez et al., 2003; Cooke, 2004). Este gusto es atribuido a una reacción adaptativa del niño en crecimiento para mantener un balance positivo de energía, aspecto que puede resultar un problema en las épocas modernas al existir una amplia y accesible oferta de alimentos densos en energía, los cuales pueden actuar como promotores de sobrepeso y obesidad en niños con hábitos inadecuados (Birch, 2000).

Otro aspecto de la percepción atribuido a la genética es la predisposición de algunos menores a percibir de manera más intensa que otros los sabores dulces y salados, al poseer una mayor densidad de papilas gustativas fungiformes en la lengua, aspecto que podría estar relacionado con ciertas preferencias, aversiones y hábitos primigenios (Keller y Tepper, 2004). Una preferencia innata en los niños por alimentos de mucha acidez (Liem y De Graff, 2004), así como aversión a los sabores picantes son tema de debate (Lawless, 1986).

La ciencia no ha establecido en forma clara la existencia de sensaciones olfativas que sean innatamente preferidas por los niños, por lo cual se plantea la hipótesis de que este tipo de preferencias son aprendidas y que se desarrollan con lentitud (Eertmans et al., 2001; Guinard, 2001).

Todas las preferencias innatas descritas anteriormente establecen una plataforma marco inicial para las futuras experiencias con los alimentos; no obstante, las mismas no necesariamente se mantienen inmutables pudiendo de hecho ser modificadas más adelante por las experiencias de consumo tempranas (Eertmans et al., 2001; Brunstrom, 2005; Menella et al., 2005).

Las preferencias y las tendencias de comportamiento son modificadas en una primera instancia por las experiencias intrauterinas que experimenta el niño (Menella, 1995; Menella y Beauchamp, 1998; Birch, 1999), lo cual tiene consecuencias a muy largo plazo (Benton, 2003). Los infantes aprenden varias preferencias por su exposición a los sabores de los alimentos de la dieta de su madre que pasan al fluido amniótico (Deakin University, 2005). Cerca de la fecha de su nacimiento se estima que un feto traga por lo menos un litro de fluido amniótico al día, exponiéndose así no sólo a sabores sino a sensaciones odoríferas (Menella y Beauchamp, 1998).

El sentido del gusto se desarrolla, entonces, activamente desde los últimos meses de la vida intrauterina alcanzando su maduración en los albores del octavo o noveno mes (Temple et al., 2002; Álvarez et al., 2003). 


\subsection{Nacimiento, lactancia y ablactación}

Desde el nacimiento y durante la infancia más inmediata, el acto de comer está principalmente motivado por el hambre más que por el deseo de subsanar alguna preferencia innata (Birch, 1999). Durante los primeros seis meses de vida, los infantes deberían consumir leche materna en exclusiva, lo cual no solo es importante desde un punto de vista nutricional, sino que la exposición a muchos de los aromas y sabores de la dieta materna que se inició en el útero, se prolonga utilizando como vehículo a la leche materna (Menella y Beauchamp, 1998).

La percepción de los sabores de la leche al iniciar la lactancia es una de las experiencias sensoriales más tempranas a las que se expone un infante neonato, y una de las de mayor impacto (Castillo et al., 2005). La escogencia del tipo de leche que se ingerirá después del nacimiento hace una gran diferencia, dado que un niño que sea alimentado con fórmula puede perderse de toda una amplia gama de estímulos sensoriales tempranos, que lo hacen propenso a aceptar más lentamente nuevos alimentos (Menella, 1995; Birch, 2000; Gerrish y Menella, 2001). La sutil variación natural diaria en el sabor de la leche materna genera también un estímulo adicional en el lactante, en contraposición con las características de sabor más estables de la fórmula nutricional (Hausner et al., 2010).

En el momento del nacimiento no sólo se inicia toda una amplia gama de estímulos nuevos derivados de la lactancia materna, si no que otras variables como el olor, el color la temperatura empieza a cobrar relevancia.

Los recién nacidos son tan sensibles a los aromas como los adultos y son capaces de percibir estímulos complejos poco después del nacimiento, como el olor de la piel de su madre y de su leche, así como una amplia gama de otros aromas (Álvarez et al., 2003; Chaluohi et al., 2005). En contraste con las preferencias por los sabores adquiridos desde el útero, las preferencias por los olores son más tardías y, por ende, más fácilmente influenciables si se busca generar un cambio más adelante empleando la exposición como mecanismo (Beauchamp y Menella, 2009).

Aunque aún hay controversia al respecto, se supone que el gusto por los colores es en su mayoría aprendido a partir del nacimiento, gracias a la influencia de las pautas y estereotipos culturales, así como por la continua exposición al entorno (Shoots, 2006). Aunque el color puede generar expectativas de sabor, ayudar a identificar sabores por asociación y tener además un fuerte efecto sensorial como un signo de rechazo o hasta 
nausea si se asocia con peligro o descomposición, no hay una teoría válida demostrada científicamente que señale en forma contundente y sin contradicciones que el color puede tener un efecto sobre la preferencia del sabor en un alimento (Garber et al., 2000).

Los niños desde el nacimiento son de hecho muy abiertos hacia el color de los alimentos, no teniendo reparos en experimentar con alimentos de colores inusuales e incongruentes (Kuntz, 1996; Garber et al., 2000). Igual situación parece presentarse cuando se trata con la temperatura, no siendo extraño que al no estar interiorizadas pautas culturales con respecto a la forma en que deben ingerirse los alimentos, los infantes ingieran gustosamente alimentos fríos que serían inaceptables para los adultos, como por ejemplo, una sopa o un panqué (Rodríguez, 2001).

A la altura del cuarto mes de vida, se empiezan a desarrollar las preferencias por el sabor salado (Menella y Beauchamp, 1998; Cooke, 2004), llegando los niños a preferir sabores salados más intensos que aquellos preferidos por los adultos, tal y como sucede con el sabor dulce (Birch, 1999; Menella et al., 2003; Benton, 2003). Los patrones más regulados con respecto al sabor salado empiezan a establecerse alrededor de los 18 a 24 meses de vida, dependiendo entonces, también, el nivel de sal aceptado del medio físico (alimento a salar) en que se presente al niño (Lawless, 1986; Menella y Beauchamp, 1998).

Alrededor del sexto mes de vida, la dieta basada únicamente en la leche materna deja de ser adecuada, haciéndose necesaria la inclusión de nuevos alimentos, proceso denominado ablactación (esto no debe dar pie a que progenitores preocupados o motivados por conceptos culturales y por la idea de que el niño debe "acostumbrar el estómago", introduzcan sólidos antes de este período) ${ }^{2},{ }^{3}$ (Benton, 2003). Debido a la transición de la dieta exclusiva en leche materna hacia la dieta omnívora, ocurre una segunda instancia de modificación de la percepción derivada del enorme aprendizaje con respecto a la comida emanado de la exposición a nuevos alimentos (Cerro et al., 2002; Birch y Fisher, 2006). En esta etapa las experiencias derivadas de la textura y la palatabilidad de los alimentos toman una alta importancia complementaria.

\footnotetext{
2 Existen estudios como el de la "Avon Longitudinal Study of Parents and Children" (ALSPAC), que señalan como la introducción de alimentos sólidos antes del cuarto mes de vida se encuentra asociada a un incremento en la obesidad infantil y en las alergias (Danowski y Gargiula, 2002; Philips, 2003).

${ }^{3}$ En el año 2002 se estimaba que menos del $5 \%$ de las madres que habitaban hogares de bajos recursos en Costa Rica alimentaban exclusivamente con leche materna a sus hijos durante los primeros 4 meses de vida (Van Esterik, 2002).
} 
La palatabilidad general es lo que se ha considerado como la fuerza motriz que estimula la selección y la preferencia temprana de un alimento en los niños (Álvarez et al., 2003). La textura influencia a los menores en la medida que afecte la facilidad con que se mastica, prefiriendo los niños aquellos alimentos fáciles de masticar (Kuntz, 1996; Rodríguez, 2001; Temple et al., 2002). Los niños tienen músculos maxilares menos desarrollados, así como una menor cantidad de dientes, aspectos que combinados resultan en una diferente percepción de la textura y en una más lenta liberación de sustancias estimulantes del gusto durante la masticación (Temple et al., 2002; Grenville et al., 2004). Las afirmaciones anteriores no deben interpretarse como una invitación para que se opte por una tardía ablactación con el afán de postergar las experiencias con la textura de los sólidos. La introducción de la dieta sólida no debe ser pospuesta por más allá de los nueve meses, pues a partir de este periodo se hace más dificultosa la aceptación de las texturas, aspecto que podría prolongarse en el tiempo y estar relacionado con rechazo de alimentos, aun a edades tan tardías como los 7 años (Coulthard et al., 2009). Los niños desde la ablactación e incluso hasta los 8 años tienden a encontrar ardua la percepción de mezclas de sabores y sobre todo de texturas (Rodríguez, 2001; Liem et al., 2004), condición que puede llevar a su rechazo (Kuntz, 1996; Oram et al., 2000).

\subsection{Primeros años de vida: etapa neofóbica, selectividad y aversión}

Después de la ablactación, y antes de sus primeros 18 meses de vida, los infantes aceptan prácticamente cualquier alimento al que se les exponga (Zeinstra et al., 2007), al punto que es común que paladeen objetos no comestibles, desagradables y hasta peligrosos, si no se les supervisa (Shutts et al., 2009). No obstante, y aunque parezca contradictorio, a estas edades se les suele exponer a pocos alimentos, debido sobre todo a preconcepciones y prejuicios (Stang, 2006). En esta etapa los niños suelen asumir que todas las personas prefieren los mismos alimentos, siéndoles imposible comprender la naturaleza subjetiva de las preferencias (Repacholi y Gopnik, 1997).

A partir del tercer o cuarto semestre de vida, los niños tienden a evitar nuevos alimentos, dándose un comportamiento denominado "neofobia" (Benton, 2003). Este fenómeno es más fuerte hacia los alimentos de origen vegetal que hacia los de origen animal (Skinner, 2000; Cooke, 2004; Nicklaus, 2009); disminuyendo si los alimentos son acentuadamente dulces o salados (Nicklaus et al., 2005c; Birch y Fisher, 2006), y 
aumentando si estos poseen una textura irregular y si no constituyen una fuente densa de energía (Birch, 1999; Nicklaus et al., 2005b).

La neofobia es un factor al que el ser humano está predispuesto genéticamente (Cooke et al., 2007; Birch, 2000), y para el que existen tantas variantes individuales en cuanto a su manifestación que puede considerársele como un rasgo de personalidad (Pliner y Salvy, 2006). Esta se incrementa con fuerza durante la niñez temprana hasta llegados los cinco años, momento a partir del cual decrece en forma paulatina hasta a los 8 años (Cooke y Wardle, 2005; Maier et al., 2007; Nicklaus, 2009). Por ello, el periodo entre la ablactación y el cuarto semestre de vida es vital para la introducción de nuevos alimentos (Nicklaus et al., 2005a).

La predisposición infantil a la neofobia podría a simple vista parecer una mala actitud (Birch, 1999), pero tiene una explicación evolutiva orientada hacia la sobrevivencia, al restringir el consumo de alimentos nuevos en una etapa donde los menores empiezan a caminar y a explorar con más independencia (Birch, 2000; Benton, 2003). El no consumir un alimento desconocido, o hacerlo en pequeñas cantidades hasta que se verifique que no causará consecuencias negativas, tiene un gran valor para la auto preservación (Maier et al., 2007), sobre todo en edades con alto riesgo de intoxicación accidental (Dagnone et al., 2002).

Entonces, se está ante un acto inconsciente que mantiene al infante en un camino alimentario "inequívoco", donde se prefieren los sabores conocidos y "seguros" (Stallberg y Pliner; 1999). La neofobia sólo existe hasta que el individuo supera el temor o disgusto de poner el alimento en su boca y paladearlo, momento a partir del cual la fobia ha sido superada en gran parte (Dovey et al., 2008). La neofobia es un constructo basado en expectativas de disgusto, apariencia visual y hasta olor en menor grado, pero no en una experiencia real con el sabor y la textura (Martins y Pliner, 2005).

No sólo la neofobia es causal de rechazo. Hay niños por naturaleza "selectivos/quisquillosos", quienes presentan un rechazo variable en cantidad y frecuencia de alimentos tanto conocidos como nuevos (Dovey et al., 2008). Es importante establecer que la neofobia y la "selectividad", aunque diferentes, son constructos relacionados (Pliner y Salvy, 2006). No se puede descartar, también, el rechazo ocasional derivado del berrinche y el capricho (Quirós, 1987). Además, hay otras causas en el rechazo de alimentos derivadas de patologías que afectan hasta un $20 \%$ de los niños entre 1 y 5 años (Gempeler et al., 
2008), y que van desde dificultades leves hasta el rechazo total de los alimentos (Sanders et al., 1993; Wright et al., 2007). Estos problemas tienen causas psicológicas como el estrés, el maltrato y el trauma; y fisiológicas como condiciones cardiopulmonares, tos crónica, colitis, alergias, anomalías anatómicas y/o neurológicas, mal absorciones, enfermedad celíaca, intolerancia, vómito persistente, reflujo, estreñimiento, dolor abdominal, gastroenteritis y en especial, los problemas con el desarrollo cognitivo e intelectual (35\% de los casos) (Gempeler et al., 2008; Kerzner, 2009; Williams et al., 2010). Los cuadros a menudo se complican, pues la resistencia de los niños invita al conflicto con los adultos, quienes en respuesta adoptan acciones coercitivas (Sanders et al., 1993).

En casos extremos los niños experimentan la denominada "aversión a los alimentos", negándose a ingerir la mayoría, si no todos, los comestibles que se le presentan, para lo cual recurren a actitudes como una conversación excesiva, la negociación, el huir de la mesa y en casos severos voltear la cabeza, lanzar la comida de la mesa, mantener la boca fuertemente cerrada, escupir, regurgitar, llorar y hasta vomitar (Werle et al., 1993; Williams et al., 2010).

Apoyo psicológico e intervención profesional se hace necesaria en estos casos (Werle et al., 1993; Williams et al., 2010), siendo útil como medida ignorar las rabietas, regresar a la boca la comida escupida, mantener la cuchara frente a la boca hasta que se acepte la comida, hacer "tiempos fuera" hasta que pase el berrinche y, en casos muy extremos, la alimentación forzada como medio para preservar la integridad física (Williams et al., 2010). Este rechazo crónico de los alimentos puede resultar en pérdida excesiva de peso, malnutrición, subdesarrollo y en casos severos, en muerte (Sanders et al., 1993; Werle et al., 1993).

La "aversión a los alimentos" no derivada de aspectos fisiológicos es usualmente asociada con la anorexia infantil, fenómeno provocado porque el niño se distrae con extrema facilidad durante las comidas, siendo incapaz de percibir las sensaciones del hambre por sobre los estímulos externos de su medio circundante. Cuando la aversión aparenta ser selectiva y supeditada a ciertos alimentos se le relaciona con aversiones sensoriales e hipersensibilidad, mientras que cuando hay manifestaciones de temor y ansiedad suele existir un desorden alimentario postraumático asociado con atragantamientos, intoxicaciones, inserción de sondas gástricas, rupturas o desprendimientos de piezas dentales, etc. (Chatoor y Ganiban, 2003). 
Las actitudes neofóbicas están evolutivamente acompañadas de mecanismos de aprendizaje asociados a la exposición, los que permiten aprender nuevas preferencias que rompen con el tiempo estas barreras (Stallberg y Pliner; 1999; Westenhoefer, 2002; Cooke et al., 2007). Las exposiciones a los alimentos reducen paulatinamente el rechazo, sobre todo cuando estas experiencias son frecuentes, y se encuentran asociadas con ambientes sociales positivos (contexto social) y con consecuencias post ingestivas agradables (Morton et al., 1999; Carruth y Skinner, 2000; Menella et al., 2006). De este modo se guarda una segura distancia con los alimentos desconocidos a medida que se experimenta poco a poco hasta llegar a la conclusión de que son seguros y aptos para ser ingeridos (Birch, 1999; Birch 2000).

Una vez superada la neofobia y la selectividad hacia un alimento, suele ser más sencillo superar las aversiones hacia otros alimentos similares, así como a la vez es más fácil introducir un alimento previamente rechazado, si se le relaciona o combina con uno ya aceptado (Birch et al., 1984; Birch, 1999). Es también digno de mencionar que en el adulto mayor es factible que se presente un resurgimiento de la neofobia, que está asociada a un sentimiento consciente de prevención de riesgos a la salud y hasta con el deterioro del aparato masticador (Dovey et al., 2008).

\subsection{Edades preescolares}

Durante los años preescolares los niños son introducidos a muchos alimentos que son más propios del mundo adulto, iniciándose la adopción de preferencias que perduran de por vida (Saint John Alderson y Ogden; 1999; Wesslen et al., 2002). Este aprendizaje, donde se empieza a asociar el gusto con las consecuencias fisiológicas y sociales de comer, lleva al desarrollo de creencias y actitudes hacia la comida que perduran incluso dominantemente durante la edad adulta (Morton et al., 1999; Benton, 2003)

Después de los 4 años, o incluso antes, la experiencia de comer trasciende la barrera de la mera satisfacción del hambre y adquiere matices influenciados por el ambiente y los factores sociales (Ramsay, 2004; Patrick y Nicklas, 2005). El entorno cultural en el que está inmerso el infante y sus padres se vuelve muy determinante, interiorizándose conceptos sobre qué, cómo, cuándo y dónde se debería comer (Birch et al., 1990; Birch, 2000; Benton, 2003). En estas edades, cuando el acto de comer adquiere matices sociales adicionales pueden imperar otros motivos de rechazo o aceptación, tales como la intuición de peligro, el 
origen y la significancia del alimento, y hasta el prejuicio (Rozin y Fallon, 1986; Benton, 2003).

Las memorias más tempranas de un niño preescolar están limitadas y supeditadas al hecho de que el infante cuente con un vocabulario tal que le permita codificar un hecho o estímulo en el momento de su ocurrencia (Bauer y Wewerka, 1995; Cain y Potts, 1996; Lumeng et al., 2005). Las desventajas socioeconómicas y la clase social puede relacionarse con la expresividad y receptividad del lenguaje en un niño, por lo cual Lumeng et al. (2005) formulan la hipótesis de que la clase social puede tener una influencia sobre la habilidad de los infantes para etiquetar las sensaciones sensoriales y, por lo tanto, su habilidad para la adquisición de una memoria por exposición que conduzca al desarrollo de preferencias. Niños de estratos sociales menos favorecidos, donde suelen existir menos periodos de estimulación temprana y de interacción infantil con los padres, pueden presentar problemas de desarrollo temprano en el lenguaje y por ende, en el desarrollo cognoscitivo en general (León, 2004).

El poder adquisitivo del grupo familiar tiene además una incidencia directa en la cantidad y en la diversidad de los alimentos a los que el niño se ve expuesto desde el nacimiento y durante toda la infancia (Bowman y Harris; 2003; Comité de Nutrición de la Sociedad Uruguaya de Pediatría, 2004, Young et al., 2004). La clase social también está muy relacionada con el nivel cultural de los padres y sus valores y, por lo tanto, con su estilo de educar a los niños, quienes establecen muchas de sus pautas de percepción de acuerdo con esta realidad (Phillips, 2003; Lumeng et al., 2005). Todo lo anterior hace de la época preescolar un período crítico en la adopción de criterios complejos de aceptación y rechazo (Nguyen y Murphy, 2003).

Los niños en esta edad son incapaces de considerar a la vez más de uno de los aspectos asociados a un estímulo (incapacidad de desglosar un objeto en sus partes constituyentes), tendiendo a aceptar lo que perciben como una realidad absoluta y a focalizarse en una sola dimensión de las cosas en detrimento de las otras (apariencia o sabor dulce por ejemplo, pero no ambas) (Guinard, 2001; Popper y Kroll, 2003; Cartín, 2005). Los valores normativos pueden no estar arraigados con fuerza en niños preescolares, por lo cual ellos no suelen clasificar los alimentos en términos de "bueno" o "malo", sobre todo en cuanto a salud se refiere (Wesslen et al., 2002). 
Uno de los aspectos que se afianza más tardíamente, y hasta incluso superada la edad preescolar, es la percepción del olor. Aunque los infantes neonatos parecen equipados con un sistema olfativo desarrollado, las respuestas afectivas rudimentarias hacia un olor no aparecen hasta una edad promedio de 5 años (Guinard, 2001; Rodríguez, 2001). Los sabores volátiles percibidos no se espera que causen un gran impacto en menores de 6 años, no debido a la imposibilidad de ser detectados, sino a que los gustos o disgustos hacia los mismos no se encuentran manifiestos con plenitud (Lawless, 1986).

Desde un punto de vista de evaluación sensorial, no es sino hasta pasados los 7 años de edad en que el olfato se ha desarrollado lo suficiente como para ser objeto de estudio, pues antes es bastante difícil establecer si las pruebas evalúan la detección del estímulo en sí o más bien la función cognoscitiva del niño (Chaluohi et al., 2005). Definir aromas es muy complicado para los menores, no sólo por su limitada familiaridad y capacidad para reconocerlos (Rodríguez, 2001; Mustonen et al., 2009), sino también porque el olfato es la modalidad sensorial cuyas sensaciones son más difíciles de describir para los niños (Noll et al., 1990; Popper y Kroll, 2003).

\section{Aspectos sociales y afectivos de la exposición a los alimentos}

\subsection{Creación de un contexto socio afectivo adecuado para la exposición}

La formación de las preferencias alimenticias en los primeros años se encuentra profundamente influenciada por el contexto cultural, socio afectivo, educativo y familiar en que los alimentos son presentados (Menella y Beauchamp, 2005; Patrick y Nicklas, 2005). Para los niños, comer es una experiencia social que les permite forjar comportamientos basándose en las observaciones que hagan del ambiente y de los demás (Birch, 1980; Birch y Fisher, 2006), haciéndose así más fácil la aceptación de un alimento (Jansen y Tenney, 2000).

La actitud de los padres en mayor instancia, y de los educadores y otros adultos hacia los nuevos alimentos, afecta en forma determinante los hábitos y comportamientos de los menores (Saint John Alderson y Ogden; 1999; Birch y Fisher, 2006; Maier et al., 2007), patrones que se establecen con firmeza ya a una edad de entre 4 a 5 años (Daniels et al., 2009). En el caso de los padres, son ellos quienes seleccionan los alimentos, el método de alimentación, el grado de control o de permisividad, y determinan a la vez los futuros roles de compra y el perfil de consumidor de sus hijos (Roedder, 1999; Birch y Fisher, 2006). En ellos 
recae también el consumo limitado de golosinas, la diversidad en la mesa, adecuados tamaños de porción, horarios de comida convenientes y la sana costumbre del desayuno entre otros (Fisher et al., 2002; Gidding et al., 2005).

La madre ostenta en casi todas las sociedades un papel preponderante, al ser su opinión la que prevalece en cuanto a qué alimentos llegan a la mesa y en qué forma estos se presentan (Benton, 2003; Liem y Menella; 2003); aspecto así reconocido por los mismos niños (Robinson, 2000). Por ello, es usual que los menores presenten afinidades sensoriales muy similares a las de su madre y a las de hermanos de edad similar (Pliner y Pelchat, 1986; Wesslen et al., 2002; Pliner y Salvy, 2006), y no tanto con el padre y con hermanos de edades y sexo diferentes (Birch y Fisher, 2000; Liem y Menella; 2003).

Lamentablemente, las exigencias modernas suelen no sólo reducir los espacios de interacción, sino que inclinan la selección nutricional hacia alimentos más convenientes y prácticos, pero a la vez más monótonos y usualmente poco nutritivos, aspecto que en ocasiones se transforma en una barrera para la exposición y las nuevas experiencias (Mustonen et al., 2009).

El ambiente de consumo establecido por los padres es una función del estilo de crianza adoptado, ya sea este permisivo (sin firmeza), autoritativo (firme, pero cariñoso y comprensivo) o bien autoritario (firme y sin contemplaciones utilizando presión y restricción al comer) (Van Strien et al., 2009). Hay una relación importante entre los estilos de crianza y el origen étnico, la clase social y la educación (Faith et al., 2003; Kroller y Warschburger, 2009). Estudios con niños mexicanos señalan que padres jóvenes y de poca educación suelen ser muy autoritarios (Arredondo et al., 2006).

La alimentación bajo un rol autoritario implica un control total e indiferente por completo a las preferencias y a las selecciones del niño, lo cual contrasta con el rol permisivo donde al infante se le permite comer las cantidades y los alimentos que desee sin que medie una estructura, siendo la disponibilidad y el deseo los únicos factores limitantes (Patrick y Nicklas, 2005). El balance ideal se logra con un estilo autoritativo, caracterizado por el soporte emocional, altos estándares de hábitos sanos, apropiada autonomía, y una clara y bidireccional comunicación (Morton et al., 1999; Joyce y Zimmer-Gembeck, 2009). Al aplicar este estilo, los padres determinan qué alimentos estarán disponibles y en qué momentos, mientras que los niños establecen cuáles serán comidos y en qué cantidades (Birch y Fisher, 2006; Hoerr et al., 2009; Van Strien et al., 2009). A pesar de sus ventajas, el estilo 
autoritativo es el que se pone en práctica de manera menos frecuente en los países desarrollados (Daniels et al., 2009).

Al aplicar un modelo permisivo, se comete el error de pensar que los niños escogerán por sí mismos alimentos saludables, efectuando estos en realidad selecciones pobres basadas en la palatabilidad y optando en consecuencia por alimentos altos en sodio, grasa y azúcar que fomentan la obesidad (Klesges et al., 1991; Hoerr et al., 2009). La mayoría del sobrepeso ganado antes de alcanzada la pubertad se obtiene alrededor de los 5 años de edad, principalmente en el caso de las niñas (91\% del peso en comparación con 75\% en los varones) (Daniels et al., 2009). Por su parte, un rol muy autoritario previene que el infante aprenda a autorregular su propia ingesta con base en la saciedad, promoviendo en cierta forma los problemas de obesidad (Benton, 2003; Hoerr et al., 2009; Joyce y ZimmerGembeck, 2009).

La alimentación de los niños parece en ocasiones errática e insuficiente a los ojos de los adultos, pero demuestra estar muy bien regulada en un período de 24 horas (Birch y Fisher, 2006). Las fluctuantes necesidades energéticas antes de los 5 años de edad y la disminución en la ingesta de varios alimentos como la leche a medida que los menores crecen, explican este fenómeno (Marín, 2000; Peña et al., 2001). Los niños y niñas no comerán un alimento que nos les guste (Birch y Fisher, 2006), mostrándose indiferentes hacia aspectos como el valor nutricional, el costo económico o la dificultad que se experimentó durante la preparación de los mismos (Birch, 1998; Soliah et al., 1997).

Ante estas realidades, quienes tutelan a los menores responden tratando de imponer un control y una presión aún mayor sobre la ingesta (Peña et al., 2001; Cooke, 2004; Powers, 2005), sobre todo en el caso de madres inexpertas acostumbradas a la mayor voracidad y poca selectividad de la infancia temprana (Marín, 2000). Esto lleva a una lucha de poder que suele alcanzar puntos álgidos durante los periodos de alimentación (Escobar, 1999; Robinson, 2000), lo que puede ser causante de trastornos emocionales y de actitudes adversas hacia los alimentos (Marín, 2000). Forzar a un menor a consumir un alimento que le desagrada violenta la preferencia del individuo, acción que lejos de incrementar la aceptación logra un poderoso efecto en la dirección opuesta (Morton et al., 1999; Benton, 2003; Ramsay, 2004). Problemas psicológicos y fisiológicos como los antes descritos en este trabajo hacen entonces su aparición (Deakin University, 2005). 
Prohibir con vehemencia un alimento es también inapropiado, pues genera hábitos muy desinhibidos cuando un menor no está bajo supervisión, provocando un alto consumo de aquellos alimentos "atractivamente prohibidos" aun en ausencia de hambre (Fisher y Birch, 1999; Fisher y Birch, 2002; Birch y Fisher, 2006), comportamiento que es considerable y acentuado en las niñas (Birch y Fisher, 2000; Fisher y Birch, 2002; Arredondo et al., 2006). Es posible que ellas sean reprimidas con más frecuencia e intensidad que los varones, dadas ciertas pautas y valores culturales de delgadez, belleza y hasta de "comer con educación", propias del concepto imperante de femineidad, razón por la cual podrían tender a "liberarse" con más ímpetu al no existir supervisión (Cullen y Zakeri, 2004; Arredondo et al., 2006). En el caso de los niños, las madres latinas tienden a asociar la imagen regordeta con un menor bien alimentado y saludable (Contento et al., 2003). La actitud controladora y hasta coercitiva que muchas madres latinas ejercen sobre la alimentación de sus niños y niñas es un hecho que se encuentra documentado en la literatura (Kaiser et al., 1999)

Un problema adicional de la restricción es que esta suele orientarse a reducir la ingesta de ciertos alimentos, pero no siempre a promover alimentos sanos y nutritivos que actúen como sustitutos, lo cual reduce el esfuerzo a un asunto de "imposición" y no de "opción" (Klesges et al., 1991; Birch y Fisher, 2006). La restricción empleada inteligentemente, no obstante, no pierde valor como técnica para reducir la ingesta energética en niños y niñas que ya tienen un cuadro de obesidad (Faith y Kerns, 2005).

Muchos adultos asumen que sus preferencias son necesariamente las mismas que las de los niños y niñas decidiendo en forma arbitraria los alimentos para los infantes sin que medie una degustación previa, lo que constituye un filtro para muchas de las experiencias sensoriales potenciales con alimentos considerados "tabú" (Amari et al., 2007). Se estructuran así ambientes donde nuevos alimentos rara vez son presentados y donde los menores no emprenden nuevas experiencias sensoriales que los lleven a superar su propia inexperiencia (Escobar, 1999; Carruth y Skinner, 2000; Álvarez et al., 2003).

Ofrecer como premio un alimento aceptado por el menor a cambio de que consuma uno que no le es de su agrado, difícilmente aumentará la predilección por este último, siendo incluso posible que la no aceptación se presente a futuro en ambos alimentos (Birch, 1999; Benton, 2003). La aceptación sí ocurre cuando el alimento es presentado en repetidas ocasiones como un premio espontáneo y no condicionado a que se consuma otro (Birch, 1999; Powers, 2005). El ofrecer un "soborno" para que se acepte un alimento no deseado, 
ha demostrado ser ineficiente como metodología para aumentar la preferencia (Birch et al., 1984; Mikula, 1989).

Intentar que un menor aumente la ingesta de un alimento insistiéndole en que el mismo es saludable, no tiene usualmente el efecto buscado; de hecho, para muchos menores la etiqueta de "saludable" suele tener una implicación hedónica desfavorable al asociarse este parámetro con un mal sabor implícito, quizás incluso reminiscente de medicamentos y hasta de procesos hospitalarios (Cooke, 2004). Los niños y niñas suelen ser expuestos con insistencia a alimentos que les desagradan y que son etiquetados como saludables, lo cual puede crear en ellos tedio (Wardle y Huon, 2000; Cooke, 2004). El introducir alimentos nuevos cuando el niño está enfermo puede ser contraproducente, al crearse asociaciones entre los alimentos y la enfermedad (Cooke et al., 2007; Aldridge et al., 2009). En todo caso, más allá que una posible implicación de mal sabor, el concepto adulto de "saludable" o "nutritivo" no tiene un valor conceptual y cognoscitivo alto entre niños y niñas menores de 10 años (Deakin University, 2005).

\subsection{Estrategias para el aumento de la aceptación}

La neofobia y la selectividad hacia los alimentos pueden ser tratados con eficacia en los niños y niñas sanas por medio de estrategias de intervención basadas en el comportamiento y la socialización que lleven a la emulación y adopción de roles por imitación (Birch, 2000; Ahearn, 2002). La modificaciones de los hábitos alimentarios se dan con mayor facilidad entre más jóvenes sean los niños y niñas, siendo de particular interés en este sentido la edad preescolar (Guinard, 2001; Phillips, 2003), durante la cual las preferencias no están todavía fuertemente arraigadas (Birch et al., 1980; Brunstrom, 2005; Lumeng et al., 2005). Una atmósfera positiva, participativa, no estresante y llena de comprensión y cariño son claves para una respuesta positiva (Escobar, 1999; Cerro et al., 2002; Benton, 2003). En este sentido, debe ser una experiencia donde los menores disfruten de autonomía y conduzcan ellos mismo el proceso hasta donde sea posible, de manera que puedan cimentar en forma paulatina sus preferencias a medida que descubren las señales propias del hambre y la saciedad (Aldridge et al., 2009). Un ambiente hostil, poco familiar y que no sea representativo de sus interacciones sociales suele ser muy estéril para lograr incrementar la aceptación sensorial de un niño o niña (Birch et al., 1984). Es por ello que ambientes como 
el hogar, el salón de clases y el comedor estudiantil son importantes, dado el gran valor afectivo, sicológico y de seguridad que tienen para los menores (Pagliarini et al., 2005).

Destacar un aspecto bueno de un alimento ("comer vegetales te hará musculoso") es más provechoso que destacar un hecho negativo ("si no comes vegetales te enfermarás") (Byrne y Nitzke, 2002). Si al degustar un sabor nuevo se provee a los niños con información positiva y semánticamente abundante sobre dicho sabor, se incrementa su habilidad para reconocer esta sensación en el futuro, a la vez que aumenta la evaluación hedónica de este estímulo (Lumeng y Cardinal, 2007), esto debido al desarrollo de una memoria y de un sentido de familiaridad con el estímulo (Lumeng y Cardinal, 2007). Los niños suelen tratar de predecir el sabor de un alimento nuevo con base en otros alimentos conocidos similares (Dovey et al., 2008). Antes de los 4 años, el efecto de la información es muy limitado dada la neofobia más acentuada que exige un mayor nivel de exposición (Cashdan, 1994).

Niños y niñas tienen una poderosa influencia en términos de la formación de criterios de aceptación y rechazo sobre otros niños con los que comparten espacios, en especial si son mayores (Birch, 1980; Warash et al., 2003), y si están entre los 3 y 5 años (Hill, 2002; Popper y Kroll, 2003). Los infantes más jóvenes suelen adoptar el gusto de los niños mayores en un esfuerzo de socialización y aceptación en el grupo (Benton, 2003; Pliner y Mann, 2004; Deakin University, 2005). Esta influencia puede ser incluso más poderosa que la influencia de los padres (Westenhoefer, 2002; Greenhalgh et al., 2009). No es necesario, de hecho, que exista un vínculo de amistad especial entre los niños para que este efecto se presente (Rozin et al., 2004). La influencia de los pares ha demostrado ser más efectiva en cuanto a cambiar las preferencias por los alimentos cuando los niños modelo tienen una actitud "socialmente agradable" más que "socialmente dominante"; además, se potencia aún más cuando el número de pares y el número de interacciones son mayores (Greenhalgh et al., 2009).

En cuanto a los adultos, los héroes intercalados en historias son mejores figuras que los adultos ordinarios (Birch, 1999; Jansen y Tenney, 2000). Paradójicamente, los maestros, si bien es cierto pueden ser modelos efectivos, sólo generan un impacto importante cuando participan de una manera entusiasta y proactiva y no cuando modelan de manera pasiva, rutinaria y desinteresada (Randenbush y Frank, 1999). El que los niños y niñas observen a su madre, su padre, su educador u otra figura patrón no sólo ofrecer, sino también comer con entusiasmo un nuevo alimento, aumenta en forma significativa las posibilidades de que 
desarrollen una buena aceptación (Carruth y Skinner, 2000; Jansen y Tenney, 2000; Benton, 2003). En este sentido particular, las madres resultan ser un gran modelo, siendo más probable que un niño se anime a consumir un alimento si observa a su madre haciéndolo (Birch, 1980; Pliner et al., 1993). Permitirles a los niños y niñas que participen en el proceso de selección, compra y preparación de los alimentos genera también resultados positivos (Warash et al., 2003).

Los individuos modelo pueden tener una influencia también negativa, la cual es en particular poderosa entre los 3 y 4 años, necesitándose sólo un individuo en un único momento para crear una aversión alimentaria difícil de superar (Greenhalgh et al., 2009), y que puede ser lo suficientemente fuerte para durar décadas (Rozin y Fallon, 1986; Benton, 2003).

A pesar de que muchos alimentos que se ofrecen a los niños y niñas suelen ser rechazados a priori por muchas de las razones antes discutidas en este documento, por medio de repetidas presentaciones en los ambientes apropiados se puede en general lograr que estos sean más propensos a ser aprobados (Ahearn, 2002; Benton, 2003; Pliner y Mann, 2004). La exposición regular inicia el proceso mediante el cual un alimento desconocido hacia el cual se pueden sentir reservas, ansiedad y hasta temor, se convierte en un alimento familiar y potencialmente preferido al lograrse una "seguridad aprendida" (Pliner y Salvy, 2006; Aldridge et al., 2009). Muchas veces los padres infieren que cuando un niño rechaza un alimento la primera vez es porque existe un genuino disgusto por el mismo, cometiéndose entonces el error de confundir con rechazo lo que es falta de familiaridad (Carruth y Skinner, 2000; Carruth et al., 2004).

El exponerse a un alimento permite diferenciar estímulos originalmente muy similares para ser discernidos, aumentando por lo tanto la habilidad para discriminar diferencias sutiles (Bauer y Wewerka, 1995; Cain y Potts, 1996; Lumeng et al., 2005). La exposición continua permite desarrollar una mayor sofisticación para establecer preferencias, empezándose a diferenciar y a apreciar estímulos que antes no eran evidentes en un alimento y que quizás lleven a desarrollar un gusto por el mismo (Young et al., 2004; Lumeng et al., 2005). Se genera en ese momento no sólo familiaridad con el gusto, sino también una familiaridad visual, asociativa y contextual (Blanchette y Brug, 2005; Mullarkey et al., 2007). La familiaridad visual es, en efecto, uno de los elementos claves al momento de que un niño prueba un nuevo alimento (Aldridge et al., 2009), lo que explica por qué puede mostrar 
preferencia por un sabor cuando se le presenta en una determinada forma física: los niños se familiarizan con los alimentos no sólo por su sabor, sino también con respecto a su nombre, apariencia, temperatura, textura y otros esquemas subjetivos (Drewnowski, 1997; Bartoshuk, 1993).

Las preferencias infantiles se forman debido a la "memoria" derivada de la experiencia de exponerse a un determinado estímulo (Álvarez et al., 2003), la que está compuesta por un nivel de "familiaridad" y un grado de "recuerdo" (Gardiner y Parkin, 1990; Gloor, 1990). Para los menores de 4 años, el reconocimiento de un alimento es, después del gusto, la dimensión más importante al aceptarlo o rechazarlo (Birch, 1980; Escobar, 1999).

Generalmente, un alimento deber ser ofrecido varias veces (de seis a 10 sesiones), en medio de contextos familiares, positivos y estimulantes antes de que sea rechazado 0 aceptado con certeza (Álvarez et al., 2003; Benton, 2003; Birch y Fisher, 2006), aunque se dan casos donde una única exposición es suficiente para lograr el efecto deseado (Reverdy et al., 2008). Para facilitar el proceso, es recomendable que la exposición continua se haga con solo un tipo de alimento a la vez (Ahearn, 2002).

Acompañar sabores desconocidos con otros que resultan atractivos (como el dulce), es una estrategia adicional para aumentar la preferencia, al crearse asociaciones placenteras por un lado y desplazar la atención de los niños por el otro (Ahearn, 2002; Havermans y Jansen, 2007; Aldridge et al., 2009).

El efecto de la exposición continuada puede ser de hecho acumulativo, pues hay evidencia de que entre más sean los alimentos que el niño aprenda a apreciar por medio de este método, más fácilmente aceptados serán otros alimentos nuevos presentados después (Greenhalgh et al., 2009).

La exposición no siempre es garantía de una mayor aceptación. En algunas ocasiones los niños pueden mantener la misma actitud aún después de este proceso, o incluso mostrar una disminución del agrado (Liem y De Graaf, 2004). En adultos, la exposición continua genera como resultado en la mayoría de los casos un decrecimiento en la aceptación, al causar tedio y aburrimiento, sentimientos de los que no están exentos algunos niños con manifiesta madurez (Aldridge et al., 2009; Liem y Zandstra, 2009). En el caso de ciertos alimentos, en especial si son muy inusuales para los niños, son necesarias más de 10 exposiciones para lograr un incremento en la preferencia (hasta 15), aspecto que resulta impráctico para procesos experimentales no sólo por los costos y el volumen de trabajo 
necesario, sino además por lo poco factible que resulta exigir la atención, la cooperación y el entusiasmo de los niños durante un periodo prolongado (Cooke y Wardle, 2005).

Una preferencia sensorial es sólo establecida por medio de todos los mecanismos antes descritos, siempre y cuando se presente un positivo sentimiento de saciedad y el consumo no sea seguido por la náusea o la enfermedad (Birch 2000; Eertmans et al., 2001; Benton, 2003). Incluso, cuando la reacción negativa no se deba directamente a lo que se ingiere, si la misma se asocia aunque sea en forma indirecta con la comida, la aversión se formará de igual forma (Birch, 1999). Existe amplia evidencia que soporta el hecho de que las aversiones condicionadas se forman de manera más rápida y perdurable hacia un alimento completamente nuevo que hacia alguno con el que ya se tenga cierta experiencia (Birch et al., 1990).

\subsection{Exposición temprana a los medios y a la cultura de consumo}

En un trabajo con niños y niñas británicos de entre 7 y 8 años, estos manifestaron con claridad que sus padres son la principal fuente de aprendizaje en lo que se refiere a alimentos (89\%), muy por encima de sus maestros (37\%) y de la televisión (31\%) (Groves, 2002).

Entre los medios de comunicación de masas, es la televisión el vehículo dominante para hacer publicidad entre niños y adolescentes, incluso por encima de otros medios importantes como la Internet (Robert Wood Johnson Foundation, 2008). En países occidentales, los infantes destinan de 21 a 22 horas semanales a ver televisión (3,3 horas al día), de las cuales 3 horas pueden corresponder a publicidad de alimentos (Powell et al., 2007a). Hasta un $50 \%$ de esos comerciales promocionan alimentos de pobre calidad nutricional y que son altos en sal, azúcar y grasa (Powell et al., 2007a; Robert Wood Johnson Foundation, 2008; Harris et al., 2009). El problema se acentúa, pues la publicidad presenta estos alimentos en un contexto positivo, feliz y socialmente aceptable (Deakin University, 2005; Allison 2009; Harris et al., 2009).

En países industrializados, hasta un $26 \%$ de la ingesta diaria de energía es consumida por los niños mientras miran la televisión (Matheson et al., 2004), inclinándose en ese momento por alimentos "chatarra", dado que permiten prestar más atención al aparato por ser de consumo fácil y rápido (Coon et al., 2001). El hábito de la televisión puede tener una relación con el sobrepeso no sólo por el consumo simultáneo de golosinas, sino porque se 
asocia con una disminución de la actividad física, un menor gasto energético basal y un menor tiempo de calidad para la interacción y modelación familiar (Olivares et al., 2003).

Los niños no comprenden la intención comercial de la publicidad, ni el concepto de costo económico y de la necesidad real de compra (Harbaugh et al., 2001). Todo lo anterior ha llevado a algunos autores a considerar que los niños menores de ocho años son una población vulnerable a la publicidad (Morton et al., 1999; Ülger, 2009). Una única exposición a un determinado anuncio televisivo puede afectar en forma considerable la preferencia de marca de un preescolar y, por ende, las percepciones sensoriales a ellas asociadas (Carruth y Skinner, 2000; Wiecha et al., 2006; Robinson et al., 2007). El estímulo asociado a las publicidad de comidas consideradas placenteras incluso puede disparar episodios de "hambre hedónica" que conducen a una necesidad de comer en ausencia de hambre real (Lowe y Butryn, 2007; Halford et al., 2008).

A partir de los 2 años, y a medida que aumenta la capacidad de verbalizar, los niños seleccionan con rapidez productos conocidos en lugar de los desconocidos, basándose exclusivamente en el atractivo o en el ideal creado por la publicidad (Matheson et al., 2004); el efecto se acentúa si se emplean colores brillantes y personajes atractivos involucrados en historias al diseñar la publicidad (Hill, 2002; Ülger, 2009). En muchos casos demandan estos productos por su nombre recurriendo a berrinches, en episodios que ocurren el $75 \%$ de las veces en el supermercado (McNeal, 1992; Robinson et al., 2007). Si bien los niños pueden reconocer marcas comerciales, en pocos casos las comprenden más allá de un nivel superficial, basando las elecciones en uno o varios atributos (tamaño, color) que facilitan el recuerdo (Roedder, 1999) y en el deseo de imitar la publicidad (Allison 2009).

Los infantes representan un gran mercado debido a la poderosa influencia que suelen tener sobre sus padres (Morton et al., 1999; Groves, 2002; Schor, 2003). Sólo en los Estados Unidos, los escolares poseían un poder adquisitivo de $\$ 30$ billones, estando en la posibilidad de influenciar hasta 10 veces más esa cifra (Wiecha et al., 2006; Powell et al., 2007b). La influencia es más profunda entre mayor sea la edad del menor, entre más numerosa sea la familia, entre más barato sea el objeto y entre mayor sea el poder adquisitivo de los padres (Roedder, 1999; Sǿndergaard y Endelenbos, 2007). 


\section{Consideraciones finales}

Los niños y niñas preescolares representan uno de los grupos de más riesgo nutricional en muchos países, donde la malnutrición no solamente afecta en forma importante la tasa de morbilidad y mortalidad, sino también el desarrollo físico e intelectual de los infantes.

En Latinoamérica son pocos los estudios orientados a la investigación de los patrones de alimentación y adopción de preferencias en niños preescolares. De esto se desprende la importancia inherente de este documento como base para futuros estudios de campo.

En Costa Rica, la ablactación de los niños es por lo general temprana, estableciéndose en promedio a los cuatro meses de edad en familias urbanas de bajos ingresos y en algunos casos hasta los 6 meses en clases sociales medias; de ellos hasta un 89\% fueron amamantados contra un $9,8 \%$ que no lo fueron (Chanto y Umaña, 1997). Llegados a la edad preescolar, se ha determinado que el componente principal de la dieta de los niños costarricenses son los lácteos en sus diversas formas, constituyéndose en la principal fuente de proteína y de grasa total (Broitman et al., 1996). Los azúcares, los cereales, así como las carnes y los huevos se encuentran también presentes en cantidades importantes, pero menores (Zúñiga, 1992), siendo fundamentales el arroz y los frijoles como "base" del plato (Maroto, 2005).

La mayoría de las actitudes, los comportamientos, las preferencias y los hábitos nutricionales hacia la comida se aprenden en los primeros años de vida por medio de un proceso complejo en el que, como se concluyó en este trabajo, media más la experiencia de cada quien que la genética. En los Estados Unidos, al menos el 30\% de la población entre los 2 y 6 años consumen frutas menos de una vez diaria, mientras $41 \%$ consume vegetales con la misma frecuencia, a la vez que ingieren con más periodicidad comidas altas en azúcares y grasas (Cooke, 2004). Mientras tanto, en Costa Rica la tendencia no es diferente, existiendo un acelerado aumento porcentual en el índice de sobrepeso en niños y niñas acompañado de un poco frecuente consumo de alimentos saludables (Morice y Achio, 2003). Este panorama hace necesario buscar opciones más saludables para paliar la ya existente pandemia de obesidad.

Lo anterior es relevante si se toma en cuenta que son los adultos, en particular las madres y los docentes, quienes juegan un rol preponderante en la formación de los roles y los patrones de alimentación. El rápido desenvolvimiento del lenguaje y la habilidad para 
aplicarlo a los alimentos durante la edad preescolar es determinante en la formación del concepto de los alimentos y, por lo tanto, los comportamientos que el infante adopta hacia ellos, haciendo de las dinámicas positivas asociadas con los alimentos tanto en el hogar como en el salón de clases una oportunidad y una necesidad. La época preescolar es un período crítico en la adopción de criterios de aceptación y rechazo, y una etapa donde los docentes encargados pueden hacer grandes diferencias formativas, al estar los preescolares en plena capacidad de aceptar cualquier alimento como una fuente nutricional si se les da la debida guía y proceso.

Muchas son las oportunidades de investigación detectadas por medio de esta revisión. Son necesarios estudios que exploren los estilos de alimentación de los preescolares costarricenses según su clase social, así como las prácticas de distribución de alimentos que tienen las madres entre sus hijos en función de su edad y género. Se requieren proyectos de investigación que comparen el efecto sobre la aceptación de productos nuevos relacionados con personajes que promuevan roles a imitar. Al respecto, existen fuertes vacíos en la literatura sobre el efecto del género y del número de estos personajes. Asimismo, es muy importante efectuar estudios más detallados que valoren comparativamente el desempeño de herramientas sensoriales en niños preescolares.

\section{Referencias}

Ahearn, William. (2002). Effect of two methods of introducing foods during feeding treatment and acceptance of previously rejected items. Behavioral Interventions, 17 (1), 111 127.

Aldridge, Victoria; Dovey, Terence M.; Halford, Jason C. (2009). The role of familiarity in dietary development. Developmental Review, 29 (1), 32-44.

Allison, Yvonne. (2009). Product Evaluation: Do the values young children attach to packaging affect their sensory evaluation of a food product and impair their objectivity? The journal of design and technology education, 4 (1), 28- 31.

Álvarez, Juliana; Cilense, Ângela Cristina.; Abreu, Fabio. (2003). Physiology of taste and food acceptance by infants. Brazilian Journal of Oral Sciences, 2 (6), 245-248.

Amari, Adrianna; Dahlquist, Lynnda; Kossoff, Eric H.; Vining, Eileen P.G.; Trescher, William H.; Slifer, Keith J. (2007). Children with seizures exhibit preferences for foods compatible with the ketogenic diet. Epilepsy y Behavior, 11 (1), 98-104. 
Arredondo, Elva M.; Elder, John P.; Ayala, Guadalupe X.; Campbell, Nadia.; Baquero, Barbara.; Duerksen, Susan. (2006). Is parenting style related to children's healthy eating and physical activity in Latino families? Health education research, 21 (6), 862871.

Bartoshuk, Linda. (1993). Distinctions between taste and smell relevant to the role of experience. En Capaldi, Elizabeth.; Powley, Terry, Taste Experience and Feeding: Development and learning (pp. 209). Washington: American Psychological Association.

Bartoshuk, Linda. (2000). Comparing Sensory Experiences across individuals: recent psychophysical advances illuminate genetic variation in taste perception. Chemical Senses, 25 (1), 447-460.

Bauer, Patricia J.; Wewerka Sandi S. (1995). One to two-year old's recall of events: the more expressed, the more impressed. Journal of Experimental Child Psychology, 59 (3), 475-496.

Beauchamp, Gary K.; Mennella, Julie A. (2009). Early flavor learning and its impact on later feeding behavior. Journal of Pediatric Gastroenterology and Nutrition, 48 (1), S25S30.

Benton, David. (2003). Role of parents in the determination of the food preferences of children and the development of obesity. International Journal of Obesity, 28 (1), 858-869.

Birch, Leann L. (1998). Psychological influences on the childhood diet. Journal of Nutrition, 128 (1), 407S-410S.

Birch, Leann L. (1999). Development of food preferences. Annual Reviews of Nutrition, 19 (1), 41- 62.

Birch, Leann L. (2000). Acquisition of Food Preferences and Eating Patterns in Children. En Harvey Anderson, H.; Blundell, J.; y Chiva, M, Food selection: From genes to culture (pp. 202). Paris: The Danone Institutes.

Birch, Leann L.; Fischer, Jennifer O. (2000). Mother's child-feeding practices influence daughter's eating and weight. American Journal of Clinical Nutrition, 71 (1), 10541061.

Birch, Leann L.; Fischer, Jennifer O. (2006). Development of eating behaviors among children and adolescents. Pediatrics, 101 (1), 539-549.

Birch, Leann Marlin, Diane W.; Rotter, Julie. (1984). Eating as the means activity in a contingency, effect on young children's food preference. Child Development, 55 (1), 431-439. 
Birch, Leann L.; McPhee, Linda.; Steinberg, Lois.; Sullivan, Susan. (1990). Conditioned flavor preferences in young children. Physiology y Behavior, 41 (1), 501-505.

Birch, Leann L.; Zimmerman, Sheryl I.; Hind, Honey. (1980). The influence of social-affective context on the formation of children's food preferences. Child Development, 51 (1), 856-861.

Birch, Leann L. (1980). Effects of peer model's food choices and eating behaviors on preschooler's food preferences. Child Development, 51 (1), 489-496.

Bowman, Shanthy A.; Harris, Ellen W. (2003). Food security, dietary choices, and televisionviewing status of preschool-aged children living in single-parent or two parent households. Family Economics and Nutrition Review, 15 (2), 29-34.

Bravo, Laura. (2004). Las destrezas perceptuales y los retos en el aprendizaje de la lectura y la escritura: una guía para la exploración y comprensión de dificultades específicas. Actualidades Investigativas en Educación, 4 (1), 1-23.

Broitman, Ingrid; Elizondo, Marilú.; León, Hannia.; León, Silvia. (1996). Patrón alimentario, densidad energética y de nutrientes de la dieta de niños menores de 3 años con retardo en el crecimiento y/o anemia ferropriva del cantón de Aserrí. Tesis para optar por el grado de Licenciatura en Nutrición Humana. Universidad de Costa Rica. San José, Costa Rica.

Brunstrom, Jeffrey M. (2005). Dietary learning in humans: directions for future research. Physiology y Behavior, 85 (1), 57-65.

Byrne, Elena; Nitzke, Susan. (2002). Preschool children's acceptance of a Novel vegetable following exposure to messages in a storybook. Journal of Nutrition Education and Behavior, 34 (1), 211-214.

Cain William S.; Potts Bonnie C. (1996). Switch and Bait: Probing the Discriminative Basis of Odor Identification via Recognition Memory. Chemical Senses, 21 (1), 35-44.

Carruth, Betty R.; Skinner, Jean D. (2000). Revisiting the picky eater phenomenon: neophobic behaviors of young children. Journal of the American College of Nutrition, 19 (6), 771-780.

Carruth, Betty R.; Ziegler, Paula J.; Gordon, Anne; Barr, Susan I. (2004). Prevalence of picky eaters among infants and toddlers and their caregivers decisions about offering a new food. Journal of the American Dietetic Association, 104 (S1), 57-64.

Cartín, Karlina. (2005). Los niños y la recepción publicitaria. Estudio sociocognitivo con niños de tres grupos de edad. Tesis para optar por el grado de Licenciatura en Psicología, Universidad de Costa Rica, San José, Costa Rica.

Cashdan, Elizabeth. 1994. A sensitive period for learning about food. Human Nature, 5 (3), 2797-291. 
Castillo, Jorge L.; Milgrom, Peter; Coldwell, Susan E.; Castillo, Ramón; Lazo, Rocío. (2005). Children's acceptance of milk with xylitol or sorbitol for dental caries prevention. Recuperado de http/www.biomedcentral.com/1472-6831/5/6

Cerro, Nadia; Zeunert, Sally; Simmer, Karen; Daniels; Lynne. (2002). Eating behavior of children $1.5-3.5$ years born preterm: parent's perceptions. Journal of Paediatrics and Child Health, 38 (1), 72-78.

Chaluohi, ChristeL; Faulcon, Patrick; Le Bihan, Christine; Hertz-Pannier, Lucie; Bonfils, Pierre; Abadie, Véronique. (2005). Olfactory evaluation in children: application to the charge syndrome. Journal of the American Academy of Pediatrics, 116 (1), 81-88.

Chanto, Silvia Elena; Umaña, Jeannina. (1997). Perfil de la alimentación de un grupo de infantes del área metropolitana de Costa Rica. Tesis para optar por el grado de Licenciatura en Nutrición Humana. Universidad de Costa Rica. San José, Costa Rica.

Chatoor, Irene; Ganiban, Jody. (2003). Food refusal by Infants and Young Children: Diagnosis and Treatment. Cognitive and Behavioral Practice, 10 (1), 138-146.

Comité de Nutrición de la Sociedad Uruguaya de Pediatría. (2004). Guías de alimentación del niño preescolar y escolar. Archivos de Pediatría del Uruguay, 75 (2), 159-163.

Contento, Isobel R.; Basch, Charles; Zybert, Patricia. (2003). Body image, weight, and food choices of Latina women and their young children. Journal of Nutrition Education Behavior, 35 (1), 236-248.

Cooke, Lucy J.; Haworth, Claire; Wardle, Jane. (2007). Genetic and environmental influences on children's food neophobia. The American Journal of Clinical Nutrition, 86 (1), 428-433.

Cooke, Lucy J.; Wardle, Jane. 2005. Age and gender differences in children's food preferences. British Journal of Nutrition, 93 (5), 741-746.

Cooke, Lucy J. (2004). The development and modification of children's eating habits. British Nutrition Foundation Nutrition Bulletin, 29 (1), 31- 33.

Coon, Katharine A.; Goldberg, Jeanne.; Rogers, Beantrice L.; Tucker, Katherine L. (2001). Relationships between use of television during meals and children's food consumption patterns. Pediatrics, 107 (1), 1-9.

Coulthard, Helen; Harris, Gillian; Emmett Pauline. (2009). Delayed introduction of lumpy foods to children during the complementary feeding period affects child's food acceptance and feeding at 7 years of age. Maternal and Child Nutrition, 5 (1),75-85.

Cullen, Karen W.; Zakeri, Issa. (2004). Fruits, vegetables, milk, and sweetened beverages consumption and access to á la Carte/snack Bar Meals at School. American Journal of Public Health, 94 (3), 463-467. 
Cullen, Karen W.; Baranowski, Tom; Rittenberry, Latroy; Olvera, Norma. (2000). Socialenvironmental influences on children's diet's: results from focus groups with African, Euro and Mexican American children and their parents. Health Education Research 15 (5), 581-590.

Dagnone, Damon; Matsui, Doreen; Rieder, Michael. (2002). Assessment of the palatability of vehicles for activated charcoal in pediatric volunteers. Pediatric Emergency Care, 18 (1), 19-21.

Daniels, Lynne A.; Magarey, Anthea; Battiustutta, Diana; Nicholson, Jan M.; Farrell, Ann; Davidson, Geoffrey; Cleghorn, Geoffrey. (2009). The NOURISH randomized control trial: Positive feeding practices and food preferences in early childhood- a primary prevention program for childhood obesity. BMC Public Health, 9 (387), 387-396.

Deakin University. (2005). Promoting healthy eating for children: a planning for practitioners. Recuperado www.health.vic.gov.au/healthpromotion/downloads/phe full.pdf

Dovey, Terence M.; Staples, Paul A.; Gibson, E. Leigh.; Halford, Jason C. (2008). Food neophobia and 'picky/fussy' eating in children: a review. Appetite, 50 (2-3), 181-193.

Drewnowski, Adam. (1997). Taste preferences and food intake. Annual Review of Nutrition, 17 (1), 237-253.

Eertmans, Audrey; Baeyens, Frank; Van Den Bergh, Omer. (2001). Food likes and their relative importance in human eating behavior: review and preliminary suggestions for health promotion. Health Education Research, 16 (4), 443- 456.

Escobar, Alyson. (1999). Factors influencing children's dietary practices: a review. Family Economics and Nutrition Review 12(1): 45-55.

Faith, Myles S.; Heshka, Stanley; Keller, Kathleen L.; Sherry, Bettylou; Matz, Patty. E.; Pietrobelli, Angelo; Allison, David B. (2003). Maternal-Child Feeding Patterns and Child Body Weight. Archives of Pediatrics y Adolescent Medicine, 157 (1), 926-932.

Faith, Myles S.; Kerns, Julia. (2005). Infant and child feeding practices and childhood overweight: the role of restriction. Maternal and Child Nutrition, 1 (1), 164-168

Fisher, Jennifer; Birch, Leann L. (1999). Restricting access to palatable foods affects children's behavioral response, food selection, and intake. American Journal Clinical Nutrition, 69 (1), 1264-1272.

Fisher, Jennifer; Birch, Leann L. (2002). Eating in the absence of hunger and overweight in girls from 5 to 7 years of age. American Journal of Clinical Nutrition, 76 (1), 226-231.

Fisher, Jennifer; Mitchell, Diane C.; Smiciklas-Wright, Helen; Birch, Leann L. (2002). Parental influences on young girl's fruit and vegetable, micronutrient, and fat intakes. Journal of the American Dietetic Association, 102 (1), 58-64. 
Garber, Lawrence.; Hyatt, Eva.; Starr, Richard G. (2000). Placing food color experimentation into a valid consumer context. Recuperado de http://www.acs.appstate.edu/ garberll/Root\%20Directory/Research/Food\%20Preferenc e/3.G,HyS01/Outline8.doc

Gardiner, John M.; Parkin, Alan J. (1990). Attention and recollective experience in recognition memory. Memory y Cognition, 18 (6), 579-583.

Gempeler, Juanita.; Rodríguez, Maritza.; Muñoz, Francisco.; Pérez, Verónica. (2008). Aversión a los alimentos en la infancia: ¿fobia, obsesión o trastornos del comportamiento alimentario no especificado? Revista Colombiana de Psiquiatría, 37 (3), 355-364.

Gerrish, Carolyn.; Mennella, Julie A. (2001). Flavor variety enhances food acceptance in formula-fed infants. American Society for Clinical Nutrition, 73 (1), 1080-1085.

Gidding, Samuel S.; Dennison, Barbara A.; Birch, Leann L.; Daniels, Stephen R.; Gilman, Matthew W.; Lichtenstein, Alice H.; Rattay, Karyl T.; Steinberger, Julia.; Stetler, Nicolas.; Van Horn, Linda. (2005). Dietary Recommendations for Children and Adolescents: A Guide for Practitioners: Consensus statement for the American Heart Association. Circulation, 112 (1), 2061-2071.

Greenhalgh, Janette.; Dowey, Alan J.; Horne, Pauline J.; Lowe, C. Fergus.; Griffiths, John H.; Whitaker, Chris J. (2009). Positive- and Negative Peer Modeling Effects on

Grenville, Rose.; Laing, David.; Oram, Nicholas.; Hutchinson, lan. (2004). Sensory profiling by children aged 6-7 and 10-11 years, part 1: a descriptor approach. Food Quality and Preference, 15 (1), 585-596.

Groves, Angela. (2002). Children's food market forces and industry responses. British Foundation Nutrition Bulletin, 27(1), 187-190.

Guinard, Jean X. (2001). Sensory and consumer testing with children. Food Science Technology, 11(1), 273- 283.

Halford, Jason C.G.; Boyland, Emma J.; Hughes, Georgina M.; Stacey, Leanne; McKean, Sara; Dovey, Terence M. (2008). Beyond brand effect of television food advertising on food choice in children: the effect of weight status. Public Health Nutrition, 11 (9), 897-904.

Harbaugh, William T.; Krause, Kate.; Berry, Timothy R. (2001). GARP for kids: On the development of Rational Choice Behavior. The American Economic Review, 91 (5), 1539-1545.

Harris, Jennifer L.; Bargh, John A.; Brownell, Kelly D. (2009). Priming Effects of Television Food Advertising on Eating Behavior. Health Psychology, 28 (4), 404-413. 
Hausner, Helene.; Nicklaus, Sophie.; Issanchou, Sylvie.; Mølgaard, Christian.; Møller, Per. (2010). Breastfeeding facilitates acceptance of a novel dietary flavour compound. Clinical Nutrition, 29 (1),141-148.

Havermans, Remco C.; Jansen, Anita. (2007). Increased children's liking of vegetables through flavour-flavour learning. Appetite, 48 (2), 259-262.

Hill, Andrew J. (2002). Developmental issues in attitudes to food and diet. Proceedings of the Nutrition Society, 61 (1), 259-266.

Hoerr, Sharon L.; Hughes, Sheryl O.; Fisher, Jennifer O.; Nicklas, Theresa A.; Liu, Yan.; Shewchuk, Richard M. (2009). Associations among parental feeding styles and children's food intake in families with limited incomes. International Journal of Behavioral Nutrition and Physical Activity, 6 (1), 55.

Jansen, Anita; Tenney, Nienke. (2000). Original communication, seeing mum drinking a light product: is social learning a stronger determinant of taste preference acquisition than caloric conditioning? European Journal of Clinical Nutrition, 55 (1), 415-422.

Joyce, Jessica L.; Zimmer-Gembeck, Melanie J. (2009). Parent feeding restriction and child weight. The mediating role of child disinhibited eating and the moderating role of the parenting context. Appetite, 782 (1), 1-9.

Kaiser, Lucia; Martinez, Nestor; Harwood, Janice; García, Linda. (1999). Child feeding strategies in low income Latino households: focus group observations. Journal of the American Dietetic Association, 99 (5), 601-603.

Keller, Kathleen; Tepper, Beverly J. (2004). Inherited taste sensitivity to 6-n-propylthiouracil in diet and body weight in children. Obesity research, 12 (6), 904-912.

Kerzner, Benny. (2009). Clinical Investigation of Feeding Difficulties in Young Children: A Practical Approach. Clinical Pediatrics, 20 (10), 1-6.

Klesges, Robert C.; Stein, Risa J.; Eck, Linda H.; Isbell, Terry R.; Klesges, Lisa M. (1991). Parental influence on food selection in young children and its relationships to childhood obesity. American Journal of Clinical Nutrition, 53 (1), 859-864.

Kroller, Katja; Warschburger, Petra. (2009). Maternal feeding strategies and child's food intake: considering weight and demographic influences using structural equation modeling. International Journal of Behavioral Nutrition and Physical Activity, 6 (1), 78-88

Kuntz, Lynn A. (1996). Designing Products for Kids. Recuperado de http://www.foodproductdesign.com/articles/466/466-0196AP.html

Lawless, Harry. (1986). Sensory development in children: Research in taste and olfaction. Journal of American Dietetic Association, 85 (1), 577-585. 
León, Ana Teresa. (2004). Desarrollo y atención del niño de 0 a 6 años: el desarrollo del niño de los dos a los seis años. San José, Costa Rica: Editorial Universidad Estatal a Distancia.

Liem, Djin G.; Graaf, Cees. (2004). Sweet and sour preferences in young children and adults: role of repeated exposure. Physiological Behavior, 83 (3), 421-429.

Liem, Djin G.; Mars, Monica; Graaf, Cees. (2004). Consistency of sensory testing with 4 and 5 year old children. Food Quality and Preference, 15 (1), 541-548.

Liem, Djin G.; Mennella, Julie A. (2003). Heightened sour preferences during childhood. Chemical Senses, 28 (1), 173-180.

Liem, Djin G.; Westerbeek, Annemarie; Wolterink, Sascha; Kok, Frans. J.; Graaf, Cees. (2004). Sour taste preferences of children relate to preference for novel and intense stimuli. Chemical Senses, 29 (1), 713-720.

Liem, Djin G.; Zandstra, Liesbeth H. (2009). Children`s liking and wanting of snack products: Influence of shape and flavour. International Journal of Behavioral Nutrition and Physical Activity, 6 (1), 38-48.

Lowe, Michael R.; Butryn, Meghan L. (2007). Hedonic hunger: a new dimension of appetite. Physiology and Behavior, 91 (4), 432-439.

Lumeng, Julie C.; Cardinal, Tiffany M. (2007). Providing Information about a Flavor to Preschoolers: Effects on Liking and Memory for Having Tasted It. Chemical Senses, 32 (1), 505-513.

Lumeng, Julie C.; Zuckerman, Matthew; Cardinal, Tiffany; Kaciroti, Niko. (2005). The association between flavor labeling and flavor recall ability in children. Chemical Senses, 30 (1), 565-574.

MacNeal, James U. (1992). Kids as consumers: a handbook of marketing to children. New York, Estados Unidos de América: Lexington Books.

Marín, Zoila R. (2000). Elementos de Nutrición Humana. San José, Costa Rica: Editorial Universidad Estatal a Distancia.

Maroto, Vanessa. (2005). Descripción de algunas características de la alimentación de niños preescolares en dos distritos de Costa Rica, 1999-2000. Tesis para optar por el grado de Licenciatura en Nutrición Humana. Escuela de Nutrición. Universidad de Costa Rica. San José, Costa Rica.

Martins,Yolanda; Pliner, Patricia. (2005). Human food choices: an examination of the factors underlying acceptance/rejection of novel and familiar animal and non animal foods. Appetite, 45 (1), 214-224. 
Matheson, Donna M.; Killen, Joel D.; Wang, Yun; Varady, Ann.; Robinson, Thomas N. (2004). Children's food consumption during television viewing. American Journal Clinical Nutrition, 79 (1), 1088-1094.

Mennella, Julie A. (1995). Mother's milk: a medium for early flavor experiences. Journal of Human Lactation, 11 (1), 39-45.

Mennella, Julie A.; Beauchamp, Gary K. (1998). Early flavor experiences: research update. Nutrition Reviews, 56 (7) 205-211.

Mennella, Julie A.; Beauchamp, Gary K. (2005). Understanding the origin of flavor preferences. Chemical Senses, 30 (1), i242-i243.

Mennella, Julie A.; Jagnow, Coren P.; Beauchamp, Gary. K. (2001). Prenatal and Postnatal Flavor Learning by Human Infants. Pediatrics, 107 (6), 1-6.

Mennella, Julie A.; Kennedy, Janice M.; Beauchamp, Gary K. (2006). Vegetable acceptance by infants: Effects of formula flavors. Early Human Development, 82 (1), 463-468.

Mennella, Julie A.; Pepino, Yanina M.; Beaucham, Gary K. (2003). Modification of bitter taste in children. Developmental Psychobiology, 43 (2), 120-127.

Mennella, Julie A.; Pepino, Yanina M.; Reed, Danielle. (2005). Genetic and environmental determinants of bitter perception and sweet preferences. Journal of the American Academy of Pediatrics, 115 (1), c216-c222.

Mikula, Gerold. (1989). Influencing food preferences of children by "if-then" type instructions. European Journal of Social Psychology, 12 (1), 225-241.

Morice, Ana; Achio, Mayra. 2003. Tendencias, costos y desafíos para la atención de las enfermedades crónicas en Costa Rica. Revista de Ciencias Administrativas y Financieras de la Seguridad Social, 11(1), 18-34.

Morton, Heather; Campbell, Karen; Santich, Barbara; Worsley, Anthony. (1999). Parental strategies and young children's snacking behavior: a pilot study. Australian Journal of Nutrition and Dietetics, 56 (4), 215-220.

Mullarkey, Dianne; Johnson, Brian; Hackett, Allan. (2007). Portion size selection of fruits and vegetables by 9 to 10 year old children in Liverpool. Journal of Human Nutrition and Dietetics, 20 (5), 459-466.

Mustonen, Sari; Rantanen, Reetta; Tuorila, Hely. (2009). Effect of sensory education on school children's food perception: A two year follow up study. Food Quality and preference, 20 (1), 230-240.

Nguyen, Simone P.; Murphy, Gregory L. (2003). An apple is more than just a fruit: cross classification in children's concepts. Child Development, 74 (6), 1783-1806.

Nicklaus, Sophie. (2009). Development of food variety in children. Appetite, 52 (1), 253-255. 
Nicklaus, Sophie; Boggio, Vincent; Chabanet, Claire; Issanchou, Sylvie. (2005a). Food choices at lunch during the third year of life: Increase in energy intake but decrease in variety. Acta Pediátrica, 94 (1), 1023-1029.

Nicklaus, Sophie; Boggio, Vincent; Chabanet, Claire; Issanchou, Sylvie. (2005c). A prospective study of food variety seeking in childhood, adolescence and early adult life. Appetite, 44 (1), 289-297.

Nicklaus, Sophie; Boggio, Vincent; Issanchou, Sylvie. (2005). Food choices at lunch during the third year of life: high selection of animal and starchy foods but avoidance of vegetables. Acta Pediátrica, 94 (7), 943-951.

Noll, Robert B.; Zucker, Robert A.; Grrenberg, Greogory S. (1990). Identification of alcohol smell among preschoolers: evidence for early socialization about drugs occurring in the home. Child Development, 61 (1), 1520-1527.

Olivares, Sonia; Yáñez, Rossana; Díaz, Nora. (2003). Publicidad de alimentos y conductas alimentarias en escolares de $5^{\circ}$ a $8^{\circ}$ básico. Revista Chilena de Nutrición, 30 (1), 3642.

Oram, Nicholas; Laing, David G.; Freeman, M. H.; Hutchinson, lan. (2000). Analysis of taste mixture by adults and children. Developmental Psychobiology, 38 (1), 67-77.

Pagliarini, Ella.; Gabbiadini, Nicola.; Ratti, Sabrina. (2005). Consumer testing with children on food combinations for school lunch. Food Quality and Preference 16 (2), 131-138.

Patrick, Heather; Nicklas, Theresa A. (2005). A review of family and social determinants of children's eating patterns and diet quality. Journal of the American College of Nutrition, 24 (2), 83-92.

Peña, Luis; Madruga, Diana; Calvo, Carmen. (2001). Alimentación del preescolar, escolar y adolescente. Situaciones especiales: dietas vegetarianas y deportes. Anales Españoles de Pediatría, 54 (5), 484-496.

Pepino, Yanina; Mennella, Julie A. (2005). Sucrose-induced analgesia is related to sweet preferences in children but not adults. Pain, 119 (1-3), 210-218.

Phillips, Frankie. (2003). Nutrition and preeschool children. British Nutrition Foundation Nutrition Bulletin, 29 (1), 64-66.

Pliner, Patricia; Mann, Nikki. (2004). Influence of social norms and palatability on amount consumed and food choice. Appetite, 42 (1), 227-237.

Pliner, Patricia; Pelchat, Marcia L. (1986). Similarities in food preferences between children and their siblings and parents. Appetite, 7 (4), 333-342.

Pliner, Patricia; Pelchat, Marcia; Grabski, Marius. (1993). Reduction of neophobia in humans by exposure to novel foods. Appetite, 20 (1), 111-122. 
Pliner, Patricia; Salvy, Sarah. (2006). Food neophobia in humans. En Shepherd, R.; Raats, M, The Psychology of Food Choice (pp. 384) Mississauga, Canadá: Cabi.

Popper, Richard; Kroll, Jeffrey. (2003). Conducting sensory research with children. Food Technology, 57(5), 60-65.

Powell, Lisa M.; Szczypka, Glen; Chaloupka, Frank J. (2007b). Exposure to food advertising on television among US children. Archives of Pediatrics y Adolescent Medicine, 161 (1),553-560.

Powell, Lisa M.; Szczypka, Glen; Chaloupka, Frank J.; Braunschweig, Carol L. (2007a). Nutritional content of television food advertisements seen by children and adolescents in the United States. Pediatrics, 120 (3),576-584.

Powers, Scott W. (2005). Parenting practices and obesity in low-income African-American preschoolers. Contractor and Cooperator Report of the United States Department of Agriculture (3), 1-16.

Quirós, Grettel. (1987). Estudio del estado nutricional de los niños, diagnóstico del servicio de alimentación y propuesta de un patrón de menú para la casa del niño hogar escuela (Guardería Infantil del Ministerio de Trabajo y Seguridad Social). Tesis para optar por el grado de Licenciatura en Nutrición Humana. Universidad de Costa Rica, San José, Costa Rica.

Ramsay, Maria. (2004). Feeding skill, appetite and feeding behaviors of infants and young children and their impact on growth and psychosocial development. Recuperado de http://www.excellence-earlychildhood.ca/documents/ramsayAGNxp.pdf

Randenbush, Bryan; Frank, Robert. (1999). Assessing food neophobia: the role of stimulus familiarity. Appetite, 32 (1), 261-271

Repacholi, Betty M.; Gopnik, Alison. (1997). Early reasoning about desires: evidence from 14 and 18 month olds. Developmental Psychology, 33 (1), 12-21.

Reverdy, Caroline; Chesnel, Florence; Schlich, Pascal; Kôster, Ep; Lange, Chris. (2008). Effect of sensory education on willingness to taste novel food in children. Appetite, 51 (1), 156-165.

Robert Wood Johnson Foundation. (2008). Food and beverage marketing to children and adolescents: What changes are needed to promote healthy eating habits? Recuperado de http://www.rwjf.org/files/research/20081103herfoodmarketing.pdf

Robinson, Sally. (2000). Children's perceptions of who controls their food. Journal of Human Nutrition and Dietetics, 13 (1), 163-171.

Robinson, Thomas N.; Borzekowski, Dina L. G.; Matheson, Donna M.; Kraemer, Helena C. (2007). Effects of fast food branding on young children's taste preferences. Archives of Pediatrics y Adolescent Medicine, 161 (8), 792-797. 
Rodríguez, Nancy. C. (2001). Developing products for children: a sensory approach. Food Product Design: Focus on Sensory. Recuperado de www.foodproductdesign.com/archive/2001/0101fo.html

Roedder, Deborah. (1999). Consumer socialization of children a retrospective look at twentyfive years of research. Journal of Consumer Research, 26 (1), 183-213.

Rozin, Paul; Fallon, April. (1986). The Acquisition of likes and dislikes for foods. Recuperado de www.nap.edu/openbook/0309036356/html/58.html

Rozin, Pau.; Riklis, Jordana; Margolis, Lara. (2004). Mutual exposure of close peer relationships does not seem to foster increased similarity in food, music or television program preferences. Appetite, 42 (1), 41-48.

Saint John Alderson, Thomas; Ogden, Jane. (1999). What do mothers feed their children and why? Health Education Research, 14 (6), 717-727.

Sanders, Matthew R.; Patel, Rinu K.; Le Grice, Bonny; Shepherd, Ross W. (1993). Children with persistent feeding difficulties: An observational analysis of the feeding interactions of problem and non-problem eaters. Health Psychology, 12 (1), 64-73.

Schor, Juliet. (2003). Work, Family and Children's consumer culture. Recuperado de http://www2.bc.edu/ schorj/consumerCulture.pdf

Shoots, Erin. (2006). The effects of stereotypes and situational factors on children's favorite and preferred colors. Recuperado de www3.interscience.wiley.com

Shutts, Kristin; Kinzler, Katherine D.; McKee, Caitlin. B.; Spelke, Elizabeth S. (2009). Social information guides infants' selection of foods. Journal of Cognition and Development, 10 (1-2), 1-17.

Soliah, Lu Ann; Walter, Janelle; Parks, Thomas; Bevill, Kathleen; Haschke, Bernadette. (1997). The role of sweeteners in the diet of young children. Early childhood education journal, 24 (4), 243-247.

Sǿndergaard, Helle A.; Edelenbos, Merete. (2007). What parents prefer and children likeinvestigating choice of vegetable-based food for children. Food Quality and Preference, 18 (7), 949-962.

Stang, Jamie. (2006). Improving the eating patterns of infants and toddlers. American Dietetic Association, 106 (1), s7-s9.

Temple, Elizabeth C.; Laing, David G.; Hutchinson, lan; Jinks, Anthony L. (2002). Temporal perception of sweetness by adults and children using computerized time-intensity measures. Chemical Senses, 27(1), 729-737.

Ülger, Billur. (2009). Packages with cartoon trade characters versus advertising: an empirical examination of preschooler's food preferences. Journal of Food Products Marketing, 15 (1), 104-117. 
Van Strien, Tatjana; Van Niekerk, Rianne; Ouwens, Machteld A. (2009). Perceived parental food controlling practices are related to obesogenic or leptogenic child life style behaviors. Appetite, 53(1), 151-154.

Warash, Bobbie; Fitch, Cindy; Bodnovich, Kara. (2003). Snack choices: helping young children make decisions. Journal of Family and Consumer Sciences, 95 (2), 60-65.

Wardle, Jane; Huon, Gail. (2000). An experimental investigation of the influence of health information on children's taste preferences. Health Education Research, 15(1), 39-44.

Werle, Mary A.; Murphy, Tria B.; Budd, Karen S. (1993). Treating chronic food refusal in young children: Home- Based parent training. Journal of Applied Behavior Analysis, 26(4), 421-433.

Wesslen, Annika; Sepp, Hanna; Fjellström, Christina. (2002). Swedish preschool children's experience of food. International Journal of Consumer Studies, 26(4), 264-271.

Westenhoefer, Joachim. (2002). Establishing dietary habits during childhood for long-term weight control. Journal of Nutrition and Metabolism, 46(1), 18-23.

Wiecha, Jean L.; Peterson, Karen E.; Ludwig, David S.; Kim, Juhee; Sobol, Arthur; Gortmaker, Steven L. (2006). When children eat what they watch: Impact of television viewing on dietary intake in youth. Archives of Pediatrics y Adolescent Medicine, 160 (1), 436-442.

Williams, Keith E.; Field, Douglas G.; Seiverling, Laura. (2010). Food refusal in children: A review of the literature. Research in Developmental Disabilities, 31 (3), 625-633.

Wright, Charlotte M.; Parkinson, Kathryn N.; Shipton, Deborah; Drewett, Robert F. (2007). How do toddler eating problems relate to their eating behavior, food preferences, and growth? Pediatrics 120, (4), e1069-e1076.

Young, Laura; Anderson, Jennifer; Beckstrom, Leslie; Bellows, Laura; Johnson, Susan. (2004). Using social marketing principles to guide development of a nutrition education initiative for preschool-aged children. Journal of Nutrition Education Behavior, 36 (1), 250-257.

Zeinstra, Gertrude G.; Koelen, Maria A.; Kok, Frans J.; De Graaf, Cees. (2007). Cognitive development and children's perceptions of fruit and vegetables; a qualitative study. Recuperado de http://www.ijbnpa.org/content/4/1/30

Zuñiga, Lauren. (1992). Descripción de la alimentación y estado nutricional de los preescolares beneficiarios de la modalidad de comidas servidas del CEN del barrio Sinaí, Pérez Zeledón. Tesis para optar por el grado de Licenciatura en Nutrición Humana. Escuela de Nutrición. Universidad de Costa Rica. San José, Costa Rica. 